\title{
Survival and Predictors of Mortality Among Adult HIV/AIDS Patients Initiating Highly Active Antiretroviral Therapy in Debre-Berhan Referral Hospital, Amhara, Ethiopia: A Retrospective Study
}

This article was published in the following Dove Press journal:

HIVIAIDS - Research and Palliative Care

\author{
Fetene Nigussie (D) \\ Abayneh Alamer $\mathbb{D}^{2}$ \\ Zuriyash Mengistu ${ }^{3}$ \\ Erdaw Tachbele $\mathbb{D}^{3}$ \\ 'Department of Nursing, College of \\ Health Science, Institute of Medicine and \\ Health Sciences, Debre Berhan \\ University, Debre Berhan, Ethiopia; \\ ${ }^{2}$ Department of Physiotherapy, College of \\ Health Sciences, Ayder Comprehensive \\ Specialized Hospital, Mekelle University, \\ Mekelle, Ethiopia; ${ }^{3}$ Department of \\ Nursing, College of Health Sciences, \\ Addis Ababa University, Addis Ababa, \\ Ethiopia
}

Background: Acquired immune deficiency syndrome is one of the most destructive epidemics the world has ever witnessed. An estimated 36.9 million people were living with HIV in 2017. HIV/AIDS is the major contributing factor for morbidity and mortality in low- and middle-income countries. Although different studies on survival and predictors of mortality among HIV/AIDS patients after initiation of antiretroviral therapy were conducted, there are inconsistencies in the findings of those studies. Furthermore, to the authors' knowledge, there was a dearth of studies conducted in this study area.

Purpose: The purpose of this study was to assess the survival and predictors of mortality among adult patients starting highly active antiretroviral therapy at Debre Berhan Referral Hospital, North Showa, Amhara, Ethiopia.

Patients and Methods: An institution-based retrospective study was conducted among the medical records of 447 study subjects' selected using simple random sampling from January 1t, 2013 to December 30, 2018. The data was collected using a structured data abstraction checklist and analyzed using Kaplan-Meier statistics and Cox regression models. Results: Among 447 adult patients, 54 patients (12.1\%) had died, giving a crude death rate of 4.18 per 100 person years $(95 \% \mathrm{CI}=3.20-5.45)$. The overall estimated survival rate after initiation of antiretroviral therapy was $81.7 \%(95 \% \mathrm{CI}=75.36-86.54 \%)$ at 72 months of follow-up. The independent predictors of mortality were clinical stage IV (HR=15.6, 95\% $\mathrm{CI}=6.609-36.948)$, baseline opportunistic infections ( $\mathrm{HR}=1.86,95 \% \mathrm{CI}=1.048-3.330)$, baseline $\mathrm{Hgb}<10 \mathrm{mg} / \mathrm{dL} \quad(\mathrm{HR}=4.655,95 \% \quad \mathrm{CI}=2.253-9.619)$, baseline $\mathrm{CD} 4<200 \quad$ cells $/ \mu \mathrm{L}$ $(\mathrm{HR}=4.71,95 \% \quad \mathrm{CI}=2.275-9.751)$, the presence of comorbidity $(\mathrm{HR}=2.56 \quad 95 \%$ $\mathrm{CI}=1.391-4.740)$, being widowed $(\mathrm{HR}=3.475,95 \% \mathrm{CI}=1.412-8.550)$, and bedridden functional status $(\mathrm{HR}=3.069,95 \% \mathrm{CI}=1.111-8.480)$.

Conclusion: Patients with opportunistic infections, advanced clinical stage disease, bedridden functional status, baseline $\mathrm{Hgb}<10 \mathrm{mg} / \mathrm{dL}$, baseline $\mathrm{CD} 4<200$ cells $/ \mu \mathrm{L}$, and comorbidity should be given special care.

Keywords: Amhara, HAART, HIV/AIDS, predictors, survival, Debre Berhan Referral Hospital

\section{Introduction}

Acquired immune deficiency syndrome (AIDS) is among the most destructive epidemics the world has ever witnessed. The United Nations program on HIV/ AIDS (UNAIDS) estimated that 36.9 million people were living with HIV (PLWH)
Correspondence: Fetene Nigussie Department of Nursing, College of Health Science, Institute of Medicine and Health Sciences, Debre Berhan University, PO Box 445, Debre Berhan, Ethiopia

Tel +25 I 932274797

Email fetenenigussie27@gmail.com 
in 2017. ${ }^{1}$ Low- and middle-income countries account for the vast majority of PLWH, with an estimated 25.5 million living in sub-Saharan Africa. Of those, 19.4 million were living in Eastern and Southern Africa, which saw 44\% of new HIV infections globally in $2016 .{ }^{2}$ HIV-related estimates and projections for Ethiopia indicate that the estimated number of adults living with HIV/AIDS was 665,116 , with an annual death rate of 12,265 in $2017 .^{3}$

By the end of 2017, 21 million people were receiving life-saving antiretroviral therapy (ART). A further 15.8 million were expected to start treatment by the WHO "treat all" recommendations to increase survival among PLWH. ${ }^{4,5}$ In 2003, the Government of Ethiopia introduced its ART program to reduce HIV-related morbidity and mortality, improve the quality-of-life of PLWH, and mitigate some of the impacts of the epidemic. ${ }^{6}$ Even though the benefit of ART for people living with HIV/ AIDS is well established in terms of improving quality-oflife and reducing morbidity and mortality, there is a regional variation in the extent of its benefit. ${ }^{7}$

Various studies which were conducted in Ethiopia showed that the estimated mortality ranged from $4.2 \%$ to $43 \%$, with the majority of deaths occurring within 6 months of initiating ART. ${ }^{8-20}$ Advanced stage of the disease, non-working functional status, low baseline hemoglobin level, lower baseline weight patients, a CD4 count $<200$ cells $/ \mu \mathrm{L}$ of blood, not taking cotrimoxazole prophylaxis therapy, opportunistic infections, educational status, place of residence, nutrition, and poor ART adherence were commonly identified as predictors of death in HIV. However, the effect of comorbidity on survival and mortality of PLWH has not been studied.

Little was also known about survival and predictors of mortality among HIV-infected patients in the study area. Hence, this study aimed to investigate the outcomes of ART, focused on adults on ART in Debre Berhan Referral Hospital, North Showa Zone, Amhara National Regional State, Ethiopia.

\section{Patients and Methods}

\section{Study Design, Area, and Period}

An institutional-based retrospective cohort study was conducted from March to April 2019 based on a medical record review of HIV/AIDS patients on HAART enrolled from January 1, 2013 to December 30, 2017, which were followed up to December 30, 2018. The study was conducted at Debre Berhan Referral Hospital, which is
$130 \mathrm{~km}$ North-East of Addis Ababa. The hospital is in Debre Berhan town, North Showa Zone, Amhara National Regional State, Ethiopia. Debre Berhan Referral Hospital was the only hospital in the North Showa area providing ART in 2007. Since the start of expanded Partnership activities like twinning with the American International Health Alliance over the years to include collaborations between Debre Berhan Referral Hospital, Mehal Meda Hospital, and Enat Hospital, and Debre Berhan University, ART uptake in the region has increased from 1,789 patients at one facility to 5,282 patients at Debre Berhan, four rural hospitals, and 86 area health centers. ${ }^{21}$ Nowadays, Debre Berhan hospital provides care for a total of 2,005 HIV/AIDS patients.

\section{Study Population}

HIV-positive adults aged 15 years or older who started ART at DBRH ART center between January 1, 2013 and December 302017 with complete intake forms, registers, and follow-up forms were the study population.

\section{Sample Size and Sampling Technique}

The sample size was determined using double population proportion formula by considering CD4 count ( $\geq 200$ cells/ $\mu \mathrm{L}$ with the proportion of 0.047 vs $<200$ cells $/ \mu \mathrm{L}$ with the proportion of 0.084 ) as an independent predictor based on a study done in Jimma university teaching hospital. ${ }^{22}$ The sample size was calculated using two-sample proportion formulas in Epi-Info version 7 for Windows. ${ }^{23}$ The calculation was based on the assumption of type I error of $5 \%$, and power of $80 \%$, providing a sample size of 447 .

A simple random sampling technique was used for selecting study subjects among 1,186 patients' cards that were obtained from the ART clinic.

\section{Study Variables}

Time to an event in months was the dependent variable. Age, sex, educational status, occupational status, marital status, CD4 count, WHO clinical staging, functional status, hemoglobin level, ART regimens and medications, risk behaviors including substance use, and adherence were the predictor variables.

\section{Data Collection}

A standard data abstraction checklist was used for recording information extracted from an electronic and paperbased database and patient cards. This form was developed 
using the standardized ART entry and follow-up form employed by the ART clinic.

The collected data was preserved in a secure environment to avoid loss and breach of confidentiality. All collected data were cleaned, validated, coded, and stored at the end of each day by the principal investigator. Processing and storing was done both electronically by entering data into epi data version 4.2.

\section{Data Quality Assurance}

The quality of data was assured before, during, and after data collection accordingly.

An objective-based and standardized questionnaire was prepared; Pretest on 5\% of medical record review was done. Data collectors and supervisors were trained. The collected data was checked for its completeness and before transferring to computer software. A non-overlapping code was given for each question and the coded data were entered and cleaned in Epi data version 4.2.

\section{Data Processing and Analysis}

Data was entered using epi data version 4.2 and Statistical analyses were performed using Stata version 15.0. Descriptive statistics such as mean, median, interquartile range (IQR), and standard deviation were used to summarize the characteristics of the cohort. A Kaplan-Meier model was used to estimate the patients` survival after ART initiation, and Log rank tests were used to compare survival curves. The necessary assumption for the model was checked by the Schoenfeld residual test.

Cox proportional hazards regression models were used to identify independent predictors of mortality and calculate hazard ratios. Bi-variable Cox proportional hazards regression was tested first and those independent variables which become fitted on the bi-variable regression at 0.25 level of significance were included in the multivariable analysis. ${ }^{24}$ Multiple Cox proportional hazards regression was done at the 0.05 level of significance to determine the net effect of each explanatory variable on time to death after initiation of ART. The results of these models were expressed as hazard ratios (HRs) with 95\% CI. STROBE cross-sectional reporting guidelines were used to assess the paper components.

\section{Results}

\section{Socio-Demographic Characteristics of the Study Participants}

Among 1,186 HIV positive adults ( $\geq 15$ years), who were enrolled from January 1, 2013 to December 30, 2018, 447 patients on ART were followed. Among 447 patients, about two-thirds of study participants were females, 358 (80.1\%) were from the urban area. The mean and median age of the cohort at ART initiation was 37 years and 36 years ( $\mathrm{IQR}=30-44$ years), with a $\mathrm{SD}$ of \pm 11 years, respectively (Table 1).

\section{Baseline Clinical, Laboratory, and ART Information of Adults on ART}

A large proportion of the study participants, that is 169 (37.7\%) of the study participants, were on WHO clinical stage II at baseline. More than three quarters (340; 76\%) of the cohort had at least one opportunistic infection. The median CD4 count was $329(\mathrm{IQR}=187-578)$ cells $/ \mu \mathrm{L}$. The median hemoglobin value was $14(\mathrm{IQR}=12.1-15.2) \mathrm{g} / \mathrm{dL}$. The vast majority (about $85 \%$ ) of patients received Cotrimoxazole prophylaxis; and $16.5 \%$ were on antituberculosis treatment (Table 2).

\section{Survival Status of Patients on ART}

The mean survival time of patients was 63.7 months $(95 \%$ $\mathrm{CI}=61.66-65.79)$ at 72 months of follow-up. The overall mortality rate in the cohort during the 1,291 person-years of observation (PYO) was 4.18 per $100 \quad(95 \%$ $\mathrm{CI}=3.20-5.45)$ person-years follow-up. The cumulative incidence of death for this study was 54 (12.1\%), with the confidence interval $(95 \% \mathrm{CI}=8.9-15.2 \%)$ of patients over 6 years. However, $393(87.9 \%)$ of the study participants were censored, including $298(66.7 \%)$ on ART, four $(0.9 \%)$ who had stopped ART, 25 (5.6\%) who were lost to follow-up, and $66(14.8 \%)$ who transferred out. More than half( $(30 ; 55.6 \%)$ of the deaths occurred during the first year of treatment initiation, while 12 $(22.2 \%)$ of the deaths occurred in the second year of follow-up.

\section{Overall Survival of Patients on ART}

In the present study, 447 patients on ART were followed up for a total of 72 months. The Kaplan-Meier survival estimation showed that the overall estimated survival rate after the initiation of HAART was $81.7 \% \quad(95 \%$ $\mathrm{CI}=75.36-86.54 \%)$ at 72 months of follow-up. The estimated cumulative survival was $92.4 \%, 89.6 \%, 88.1 \%$, $86.6 \%, 85.3 \%$, and $81.7 \%$ at $12,24,36,48,60$, and 72 months, respectively (Figure 1). The study revealed that the highest rate of mortality occurred during the first year of initiation of HAART. 
Table I Baseline Socio-Demographic Characteristics of Adult HIVIAIDS Patients on ART in DBRH ART Clinic, North Showa, Amhara National Regional State, Ethiopia, $2019(n=447)$

\begin{tabular}{|c|c|c|c|c|}
\hline \multirow[t]{2}{*}{ Variables } & \multirow[t]{2}{*}{ Category } & \multicolumn{2}{|c|}{$\begin{array}{l}\text { Vital Status at Last } \\
\text { Contact }\end{array}$} & \multirow[t]{2}{*}{$\begin{array}{l}\text { Total, } \\
\text { n (\%) }\end{array}$} \\
\hline & & $\begin{array}{l}\text { Censored, } \\
\text { n (\%) }\end{array}$ & $\begin{array}{l}\text { Death, } \\
\text { n (\%) }\end{array}$ & \\
\hline Age of patient & $\begin{array}{l}15-24 \\
25-34 \\
35-44 \\
\geq 45\end{array}$ & $\begin{array}{l}4 \mid(95.35) \\
|3|(89.12) \\
122(83.56) \\
99(89.19)\end{array}$ & $\begin{array}{l}2(4.65) \\
16(10.88) \\
24(16.44) \\
12(10.81)\end{array}$ & $\begin{array}{l}43(9.62) \\
147(32.89) \\
146(32.66) \\
111(24.83)\end{array}$ \\
\hline Sex & $\begin{array}{l}\text { Male } \\
\text { Female }\end{array}$ & $\begin{array}{l}129(87.16) \\
264(88.29)\end{array}$ & $\begin{array}{l}19(12.84) \\
35(11.71)\end{array}$ & $\begin{array}{l}148(33.11) \\
299(66.89)\end{array}$ \\
\hline Family size & $\begin{array}{l}\leq 2 \\
3-4 \\
>4\end{array}$ & $\begin{array}{l}107(88.43) \\
165(85.05) \\
121(91.67)\end{array}$ & $\begin{array}{l}14 \text { (II.57) } \\
29(14.95) \\
11(8.33)\end{array}$ & $\begin{array}{l}121(27.1) \\
194(43.4) \\
132(29.5)\end{array}$ \\
\hline Region & $\begin{array}{l}\text { Amhara } \\
\text { Oromia } \\
\text { Others }\end{array}$ & $\begin{array}{l}364(87.9) \\
22(84.6) \\
7\end{array}$ & $\begin{array}{l}50(12.1) \\
4(15.4)\end{array}$ & $\begin{array}{l}4 \mid 4(92.6) \\
26(5.8) \\
7(1.6)\end{array}$ \\
\hline $\begin{array}{l}\text { Residence of } \\
\text { patients }\end{array}$ & $\begin{array}{l}\text { Urban } \\
\text { Rural }\end{array}$ & $\begin{array}{l}312(87.15) \\
81(91.01)\end{array}$ & $\begin{array}{l}46(12.85) \\
8(8.99)\end{array}$ & $\begin{array}{l}358(80.1) \\
89(19.9)\end{array}$ \\
\hline $\begin{array}{l}\text { Marital status of } \\
\text { women }\end{array}$ & $\begin{array}{l}\text { Single } \\
\text { Married } \\
\text { Divorced } \\
\text { Widowed }\end{array}$ & $\begin{array}{l}82(91.1) \\
224(89.6) \\
43(76.8) \\
44(86.27)\end{array}$ & $\begin{array}{l}8(8.9) \\
26(10.4) \\
13(23.2) \\
7(13.73)\end{array}$ & $\begin{array}{l}90(20.13) \\
250(55.92) \\
56(12.53) \\
51(11.42)\end{array}$ \\
\hline $\begin{array}{l}\text { Educational } \\
\text { status }\end{array}$ & $\begin{array}{l}\text { I. Illiterate } \\
\text { I. Can read and } \\
\text { write } \\
\text { I. Primary } \\
\text { I. Secondary } \\
\text { Tertiary }\end{array}$ & $\begin{array}{l}90(87.4) \\
83(89.25) \\
113(89.7) \\
69(86.25) \\
38(84.4)\end{array}$ & $\begin{array}{l}13(12.6) \\
10(10.75) \\
13(10.3) \\
11(13.75) \\
7(15.6)\end{array}$ & $\begin{array}{l}103(23) \\
93(20.8) \\
126(28.2) \\
80(17.9) \\
45(10.1)\end{array}$ \\
\hline $\begin{array}{l}\text { Occupational } \\
\text { status }\end{array}$ & $\begin{array}{l}\text { Farmer } \\
\text { I. Merchant } \\
\text { Governmental } \\
\text { employee } \\
\text { Non-governmental } \\
\text { employee } \\
\text { Day laborer } \\
\text { Jobless } \\
\text { Driver } \\
\text { Housewife }\end{array}$ & $\begin{array}{l}39(86.7) \\
44(91.7) \\
59(86.76) \\
48(87.27) \\
42(84) \\
52(89.66) \\
4(100) \\
105(88.24)\end{array}$ & $\begin{array}{l}6(13.3) \\
4(8.3) \\
9(13.24) \\
7(12.73) \\
8(16) \\
6(10.34) \\
0 \\
14(11.76)\end{array}$ & $\begin{array}{l}45(10.1) \\
48(10.7) \\
68(15.2) \\
55(12.3) \\
50(11.2) \\
58(13) \\
40.9) \\
119(26.6)\end{array}$ \\
\hline $\begin{array}{l}\text { Disclosure } \\
\text { status }\end{array}$ & $\begin{array}{l}\text { Disclosed } \\
\text { Not disclosed }\end{array}$ & $\begin{array}{l}328(89.37) \\
6581.25)\end{array}$ & $\begin{array}{l}39(10.63) \\
15(18.75)\end{array}$ & $\begin{array}{l}367(82.1) \\
80(17.9)\end{array}$ \\
\hline
\end{tabular}

\section{Survival Function Among Different Groups of Patients on ART}

The Log rank test was conducted to check for the existence of any significant differences in survival among the various levels of categorical variables considered in the
Table 2 Baseline Clinical, Laboratory, and Treatment-Related Characteristics of Adult HIV/AIDS Patients on ART in DBRH ART Clinic, North Showa, Amhara National Regional State, Ethiopia, $2019(n=447)$

\begin{tabular}{|c|c|c|c|c|}
\hline \multirow[t]{2}{*}{ Variables } & \multirow[t]{2}{*}{ Category } & \multicolumn{2}{|c|}{$\begin{array}{l}\text { Vital Status at Last } \\
\text { Contact }\end{array}$} & \multirow[t]{2}{*}{$\begin{array}{l}\text { Total, } \\
\text { n (\%) }\end{array}$} \\
\hline & & $\begin{array}{l}\text { Censored, } \\
\mathrm{n}(\%)\end{array}$ & $\begin{array}{l}\text { Death, } \\
\text { n (\%) }\end{array}$ & \\
\hline WHO staging & $\begin{array}{l}\text { Stage I } \\
\text { Stage II } \\
\text { Stage III } \\
\text { Stage IV }\end{array}$ & $\begin{array}{l}149(94.3) \\
153(90.5) \\
76(85.4) \\
15(48.4)\end{array}$ & $\begin{array}{l}9(5.7) \\
16(9.5) \\
13(14.5) \\
16(51.6)\end{array}$ & $\begin{array}{l}158(35.3) \\
169(37.8) \\
89(19.9) \\
31(7)\end{array}$ \\
\hline $\begin{array}{l}\text { Opportunistic } \\
\text { infections }\end{array}$ & $\begin{array}{l}\text { No } \\
\text { Yes }\end{array}$ & $\begin{array}{l}83(77.57) \\
310(91.2)\end{array}$ & $\begin{array}{l}24(22.43) \\
30(8.8)\end{array}$ & $\begin{array}{l}107(24) \\
340(76)\end{array}$ \\
\hline $\begin{array}{l}\text { TB treatment } \\
\text { during follow- } \\
\text { up }\end{array}$ & $\begin{array}{l}\text { Yes } \\
\text { No }\end{array}$ & $\begin{array}{l}57(77) \\
336(90.1)\end{array}$ & $\begin{array}{l}17(23) \\
37(9.9)\end{array}$ & $\begin{array}{l}74(16.5) \\
373(83.5)\end{array}$ \\
\hline CD4 count & $\begin{array}{l}\geq 200 \text { cells } / \mu \mathrm{L} \\
<200 \text { cells } / \mu \mathrm{L}\end{array}$ & $\begin{array}{l}304(96.2) \\
89(67.9)\end{array}$ & $\begin{array}{l}12(3.8) \\
42(32.1)\end{array}$ & $\begin{array}{l}316(70.7) \\
131(29.3)\end{array}$ \\
\hline $\begin{array}{l}\text { Body mass } \\
\text { index }\end{array}$ & $\begin{array}{l}\leq 18.5 \\
18.5-25 \\
\geq 25\end{array}$ & $\begin{array}{l}82(67.8) \\
239(91.6) \\
62(95.4)\end{array}$ & $\begin{array}{l}29(32.2) \\
22(8.4) \\
3(4.6)\end{array}$ & $\begin{array}{l}12 \mid(27.1) \\
26 \mid(58.4) \\
65(14.5)\end{array}$ \\
\hline $\begin{array}{l}\text { Functional } \\
\text { status at base } \\
\text { line }\end{array}$ & $\begin{array}{l}\text { Working } \\
\text { Ambulatory } \\
\text { Bedridden }\end{array}$ & $\begin{array}{l}325(92.9) \\
67(77.9) \\
I(9.1)\end{array}$ & $\begin{array}{l}25(7.1) \\
19(22.1) \\
10(90.9)\end{array}$ & $\begin{array}{l}350(78.3) \\
86(19.2) \\
11(2.5)\end{array}$ \\
\hline $\begin{array}{l}\text { Hgb at base } \\
\text { line }\end{array}$ & $\begin{array}{l}\geq 10 \mathrm{gm} / \mathrm{dL} \\
<10 \mathrm{gm} / \mathrm{dL}\end{array}$ & $\begin{array}{l}365(93.8) \\
28(48.3)\end{array}$ & $\begin{array}{l}24(6.2) \\
30(51.7)\end{array}$ & $\begin{array}{l}389(87) \\
58(13)\end{array}$ \\
\hline $\begin{array}{l}\text { ART eligibility } \\
\text { criteria }\end{array}$ & $\begin{array}{l}\text { CD4 below } 200 \\
\text { WHO stage I, II, } \\
\text { and III with TLC } \\
<1,200 \\
\text { WHO stage IV } \\
\text { Residence of } \\
\text { catchment area } \\
\text { No identified } \\
\text { barriers for } \\
\text { adherence }\end{array}$ & $\begin{array}{l}205(87.6) \\
85(98.8) \\
32(68.1) \\
28(93.3) \\
43(86)\end{array}$ & $\begin{array}{l}29(12.4) \\
1(1.2) \\
15(31.9) \\
2(6.7) \\
7(14)\end{array}$ & $\begin{array}{l}234(52.4) \\
86(19.2) \\
47(10.5) \\
30(6.7) \\
50(11.2)\end{array}$ \\
\hline CPT & $\begin{array}{l}\text { Yes } \\
\text { No }\end{array}$ & $\begin{array}{l}341(89.3) \\
52(77.6)\end{array}$ & $\begin{array}{l}39(10.7) \\
15(22.4)\end{array}$ & $\begin{array}{l}380(85) \\
67(15)\end{array}$ \\
\hline Comorbidity & $\begin{array}{l}\text { Yes } \\
\text { No }\end{array}$ & $\begin{array}{l}59(65.6) \\
334(93.6)\end{array}$ & $\begin{array}{l}31(34.4) \\
23(6.4)\end{array}$ & $\begin{array}{l}90(20.1) \\
357(79.9)\end{array}$ \\
\hline Substance use & $\begin{array}{l}\text { Yes } \\
\text { No }\end{array}$ & $\begin{array}{l}96(82.8) \\
297(89.7)\end{array}$ & $\begin{array}{l}20(17.2) \\
34(10.3)\end{array}$ & $\begin{array}{l}116(26) \\
331(74)\end{array}$ \\
\hline Adherence & $\begin{array}{l}\text { Good } \\
\text { Fair } \\
\text { Poor }\end{array}$ & $\begin{array}{l}373(90.5) \\
17(65.4) \\
3(33.3)\end{array}$ & $\begin{array}{l}39(9.5) \\
9(34.6) \\
6(66.7)\end{array}$ & $\begin{array}{l}412(92.2) \\
26(5.8) \\
9(2)\end{array}$ \\
\hline
\end{tabular}

Abbreviations: ART, antiretroviral therapy; CD4, cluster for differentiation; CPT, cotrimoxazole prophylaxis therapy; Hgb, hemoglobin; TB, tuberculosis; TLC, total lymphocyte count; WHO, World Health Organization. 


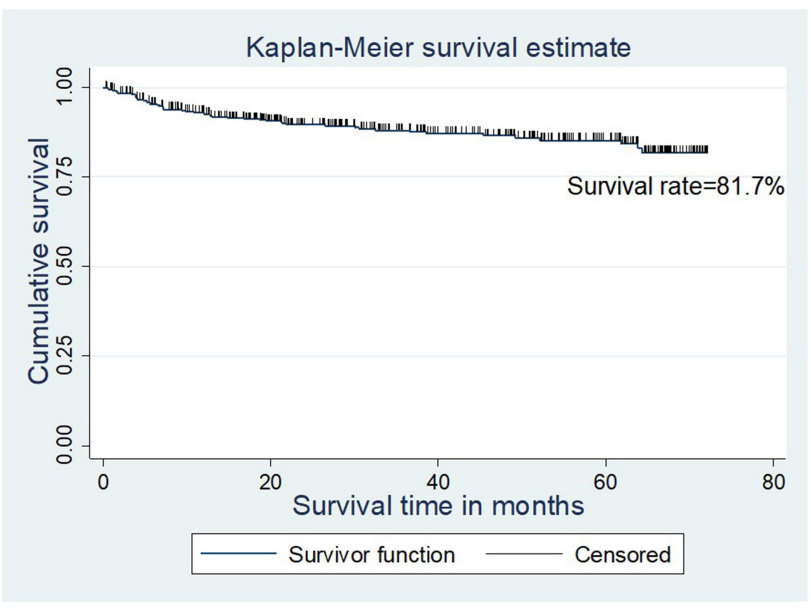

Figure I Overall Kaplan-Meier estimates of patients starting ART in DBRH from January I, 2013 to December 30, 2018.

study. The test statistics in this study showed significant associations in survival function for different categorical variables. Accordingly, the Kaplan-Meier analysis indicated significant evidence of differences in baseline opportunistic infections, functional status, comorbidity, WHO clinical staging, baseline hemoglobin, and baseline CD4 count. The Kaplan-Meier survival function indicated that those who had WHO clinical stage I, II, and III at baseline had a longer survival time than those with WHO clinical stage IV (34.3 months, 95\% $\mathrm{CI}=23.03-45.6)$. This difference was statistically significant at $P<0.0001$. The mean survival time for those who had comorbidity and past opportunistic infection was 49.13 months $(95 \% \mathrm{CI}=42.70-55.56)$ and 57.1 months (95\% CI=51.8-62.4), respectively. The survival time of patients with bedridden functional status was shorter than those with working and ambulatory functional status (7.26 months, 95\% CI=3.75-10.76). This difference was statistically significant at $P<0.0001$.

The lowest survival probabilities were observed for patients with a bedridden functional status $(0 \%)$, baseline hemoglobin $<10$ (36.74\%), a baseline CD4 count $<200$ cells/ $\mu \mathrm{L}$ (55.25\%), and WHO stage IV disease (45.77\%) (Table 3).

Figure 2 shows that those who had no baseline opportunistic infections had better mean survival time, which was 65.84 months $(95 \% \mathrm{CI}=63.75-67.93)$, than those who had opportunistic infections at the baseline (57.1 months, 95\% CI $=51.8-62.4)$, this difference was statistically significant with $P<0.001$.

The mean survival time for those who had clinical stage I, II, or III at baseline was longer than those in advanced clinical stage (IV) (34.3 months, 95\% $\mathrm{CI}=23.03-45.6)$; this difference was statistically significant at $P<0.0001$ (Figure 3).

The Kaplan-Meier graph shows that mean survival time for those who had working and ambulatory functional status was 67.25 months $(95 \% \mathrm{CI}=65.46-69.05)$ for working and 56.41 months $(95 \% \mathrm{CI}=50.37-62.45)$ for ambulatory, which was higher than the mean survival time of bedridden individuals (7.26 months, 95\% $\mathrm{CI}=3.75-10.76)$. This difference was statistically significant at $P<0.0001$ (Figure 4).

The Kaplan-Meier graph shows that the mean survival time for those who had hemoglobin level $\geq 10 \mathrm{mg} / \mathrm{dL}$ was 67.81 months $(95 \% \mathrm{CI}=66.19-69.43)$, which was higher than the mean survival time of individuals who had a hemoglobin level below $10 \mathrm{mg} / \mathrm{dL}$ (36.74 months, 95\% $\mathrm{CI}=28.3-45.2)$. This difference was statistically significant at $P<0.01$ (Figure 5).

Regarding the CD4 count, the mean survival time for those who had a CD4 count $\geq 200$ cells $/ \mu \mathrm{L}$ had a longer survival time than those with a CD4 count $<200$ cells $/ \mu \mathrm{L} \quad(50.14$ months, 95\% CI=44.8-55.5), which was statistically significant at $P<0.0001$ (Figure 6).

The Kaplan-Meier graph along with the Log rank test revealed that the mean survival time for those who had no comorbidity was longer (67.62 months, 95\% CI=65.89-69.35) than those who had comorbidity (49.13 months, 95\% CI=42.70-55.56). This difference was statistically significant at $P<0.0001$ (Figure 7)

\section{Predictors of Mortality}

In the bivariable Cox Proportional Hazard regression model, marital status, disclosure status, substance use, baseline opportunistic infections, WHO clinical stage, functional status, baseline hemoglobin level, baseline CD4 count, body mass index, Cotrimoxazole prophylactic therapy adherence, and comorbidity were all associated with survival status $(P<0.25)$.

In multivariable Cox regression analysis, those variables with a $P$-value $<0.25$ in the bivariable analysis were included. In the multivariable Cox proportional hazards model, seven variables were associated with the mortality of patients on ART.

The result of the multivariable analysis revealed that those who had opportunistic infection had a 1.86-times higher chance of death compared to those who had not $(\mathrm{aHR}=1.86,95 \% \mathrm{CI}=1.048-3.33)$. Patients who had 
Table 3 Survival Time, Cumulative Survival Probability, Significance, and Log Rank Test for the Study Population According to Different Characteristics of Patients During 6 Years of Follow-UP (Kaplan-Meier Method) of HIVIAIDS Patients on ART in DBRH ART Clinic, North Showa, Amhara National Regional State, Ethiopia, $2019(n=447)$

\begin{tabular}{|c|c|c|c|c|}
\hline Variables & Category & $\begin{array}{l}\text { Mean Survival Time in } \\
\text { Months }(95 \% \mathrm{Cl})\end{array}$ & $\begin{array}{l}\text { Overall, 6-Year } \\
\text { Survival (\%) }\end{array}$ & $\begin{array}{l}\text { Log Rank Test } \\
\text { (P-value) }\end{array}$ \\
\hline Age of patient & $\begin{array}{l}15-24 \\
25-34 \\
35-44 \\
\geq 45\end{array}$ & $\begin{array}{l}68.64(64.131-73.2) \\
64.68(61.4-67.98) \\
60.6(56.46-64.74) \\
64.27(60.29-68.24)\end{array}$ & $\begin{array}{l}93.8 \\
79.79 \\
75.4 \\
87.2\end{array}$ & 0.1301 \\
\hline Sex & $\begin{array}{l}\text { Male } \\
\text { Female }\end{array}$ & $\begin{array}{l}63.05(59.31-66.79) \\
63.90(61.40-66.40)\end{array}$ & $\begin{array}{l}84.92 \\
78.81\end{array}$ & 0.8217 \\
\hline Residence & $\begin{array}{l}\text { Urban } \\
\text { Rural }\end{array}$ & $\begin{array}{l}63.30(60.90-65.06) \\
65.54(61.28-69.80)\end{array}$ & $\begin{array}{l}79.78 \\
90.09\end{array}$ & 0.3909 \\
\hline Religion & $\begin{array}{l}\text { Orthodox } \\
\text { Muslim } \\
\text { Protestant } \\
\text { Others }\end{array}$ & $\begin{array}{l}63.70(61.49-65.92) \\
65.478(60.00-70.95) \\
59.35(45.6-73.09) \\
46.92(12.2-81.58)\end{array}$ & $\begin{array}{l}82.12 \\
80.19 \\
81.82 \\
50\end{array}$ & 0.6106 \\
\hline Educational status & $\begin{array}{l}\text { Illiterate } \\
\text { Can read and write } \\
\text { Primary } \\
\text { Secondary } \\
\text { Tertiary }\end{array}$ & $\begin{array}{l}63.88(59.88-67.89) \\
64.48(60.09-68.87) \\
64.60(60.90-68.42) \\
62.64(57.60-67.67) \\
59.33(51.86-66.79)\end{array}$ & $\begin{array}{l}76.15 \\
85.83 \\
86.78 \\
82.11 \\
71.67\end{array}$ & $1.70(0.7899)$ \\
\hline Occupational statues & $\begin{array}{l}\text { Farmer } \\
\text { Merchant } \\
\text { Governmental employee } \\
\text { Non-governmental } \\
\text { employee } \\
\text { Day laborer } \\
\text { Jobless } \\
\text { Housewife }\end{array}$ & $\begin{array}{l}61.10(54.18-68.03) \\
65.80(60.04-71.57) \\
62.13(56.30-68.02) \\
62.58(56.16-69.00) \\
61.40(54.70-68.11) \\
65.65(61.03-70.27) \\
64.19(60.40-67.97)\end{array}$ & $\begin{array}{l}85.08 \\
89.09 \\
84.46 \\
80.36 \\
\\
79.39 \\
72.28 \\
81.76\end{array}$ & $2.31(0.9409)$ \\
\hline Family size & $\begin{array}{l}\leq 2 \\
3-4 \\
\geq 4\end{array}$ & $\begin{array}{l}64.33(60.52-68.13) \\
61.89(58.52-65.26) \\
65.91(62.64-69.20)\end{array}$ & $\begin{array}{l}78.64 \\
79.56 \\
89.95\end{array}$ & $2.65(0.2662)$ \\
\hline Marital status & $\begin{array}{l}\text { Married } \\
\text { Single } \\
\text { Widowed } \\
\text { Divorced }\end{array}$ & $\begin{array}{l}65.76(61.67-69.86) \\
64.82(62.24-67.40) \\
62.16(55.45-68.87) \\
56.25(49.12-63.40)\end{array}$ & $\begin{array}{l}88.26 \\
85.32 \\
80.52 \\
50.89\end{array}$ & $8.62(<0.05)$ \\
\hline Disclosure status & $\begin{array}{l}\text { Disclosed } \\
\text { Not disclosed }\end{array}$ & $\begin{array}{l}64.60(62.40-66.78) \\
59.68(54.05-65.33)\end{array}$ & $\begin{array}{l}84.55 \\
69.59\end{array}$ & $3.88(<0.05)$ \\
\hline WHO staging & $\begin{array}{l}\text { Stage I } \\
\text { I. Stage II } \\
\text { Stage III } \\
\text { Stage IV }\end{array}$ & $\begin{array}{l}68.14(65.70-70.59) \\
66.08(63.32-68.84) \\
59.75(53.99-65.50) \\
34.30(23.03-45.60)\end{array}$ & $\begin{array}{l}92.89 \\
80.29 \\
77.20 \\
45.77\end{array}$ & $88.49(<0.0001)$ \\
\hline Opportunistic infections & $\begin{array}{l}\text { Yes } \\
\text { No }\end{array}$ & $\begin{array}{l}57.10(51.80-62.40) \\
65.84(63.75-67.93)\end{array}$ & $\begin{array}{l}71.86 \\
85.02\end{array}$ & $12.90(<0.001)$ \\
\hline $\begin{array}{l}\text { TB treatment during } \\
\text { follow-up }\end{array}$ & $\begin{array}{l}\text { Yes } \\
\text { No }\end{array}$ & $\begin{array}{l}56.11(49.50-62.71) \\
65.19(63.12-67.26)\end{array}$ & $\begin{array}{l}74.11 \\
82.87\end{array}$ & $10.18(0.001)$ \\
\hline
\end{tabular}

(Continued) 
Table 3 (Continued).

\begin{tabular}{|c|c|c|c|c|}
\hline Variables & Category & $\begin{array}{l}\text { Mean Survival Time in } \\
\text { Months }(95 \% \mathrm{Cl})\end{array}$ & $\begin{array}{l}\text { Overall, 6-Year } \\
\text { Survival (\%) }\end{array}$ & $\begin{array}{l}\text { Log Rank Test } \\
(P \text {-value) }\end{array}$ \\
\hline Comorbidity & $\begin{array}{l}\text { Yes } \\
\text { No }\end{array}$ & $\begin{array}{l}49.13(42.70-55.56) \\
67.62(65.89-69.35)\end{array}$ & $\begin{array}{l}58.42 \\
87.90\end{array}$ & $54.53(<0.0001)$ \\
\hline CD4 count & $\begin{array}{l}\geq 200 \text { cells } / \mu \mathrm{L} \\
<200 \text { cells } / \mu \mathrm{L}\end{array}$ & $\begin{array}{l}69.38(67.93-70.83) \\
50.14(44.79-55.50)\end{array}$ & $\begin{array}{l}92.80 \\
55.25\end{array}$ & $77.23(<0.0001)$ \\
\hline Body mass index & $\begin{array}{l}\leq 18.5 \\
18.5-25 \\
\geq 25\end{array}$ & $\begin{array}{l}54.90(49.58-60.22) \\
66.27(63.98-68.56) \\
68.87(65.49-72.25)\end{array}$ & $\begin{array}{l}67.16 \\
84.24 \\
94.86\end{array}$ & $26.95(<0.0001)$ \\
\hline $\begin{array}{l}\text { Functional status at base } \\
\text { line }\end{array}$ & $\begin{array}{l}\text { Working } \\
\text { Ambulatory } \\
\text { Bedridden }\end{array}$ & $\begin{array}{l}67.25(65.46-69.05) \\
56.41(50.37-62.45) \\
7.26(3.75-10.76)\end{array}$ & $\begin{array}{l}86.94 \\
71.42 \\
0.000\end{array}$ & $201.57(<0.0001)$ \\
\hline $\mathrm{Hgb}$ at base line & $\begin{array}{l}\geq 10 \mathrm{gm} / \mathrm{dL} \\
<10 \mathrm{gm} / \mathrm{dL}\end{array}$ & $\begin{array}{l}67.81(66.19-69.43) \\
36.74(28.33-45.15)\end{array}$ & $\begin{array}{l}88.91 \\
34.90\end{array}$ & $128.58(<0.000 \mid)$ \\
\hline CPT & $\begin{array}{l}\text { Yes } \\
\text { No }\end{array}$ & $\begin{array}{l}64.96(62.87-67.04) \\
56.55(49.83-63.27)\end{array}$ & $\begin{array}{l}84.14 \\
67.58\end{array}$ & $8.13(<0.01)$ \\
\hline Substance use & $\begin{array}{l}\text { Yes } \\
\text { No }\end{array}$ & $\begin{array}{l}60.39(55.78-64.90) \\
64.80(62.63-67.14)\end{array}$ & $\begin{array}{l}71.16 \\
85.47\end{array}$ & $4.03(<0.05)$ \\
\hline Adherence & $\begin{array}{l}\text { Good } \\
\text { Fair } \\
\text { Poor }\end{array}$ & $\begin{array}{l}65.49(63.50-67.40) \\
45.20(34.79-55.61) \\
29.73(13.95-45.52)\end{array}$ & $\begin{array}{l}85.38 \\
34.33 \\
25.40\end{array}$ & $49.03(<0.0001)$ \\
\hline
\end{tabular}

comorbidity had a 2.56-times higher risk of dying than those who did not have comorbidity $(\mathrm{aHR}=2.56$, 95\% $\mathrm{CI}=1.39-4.74)$. Regarding WHO clinical staging, patients with stage III had a 2.88-times higher risk of

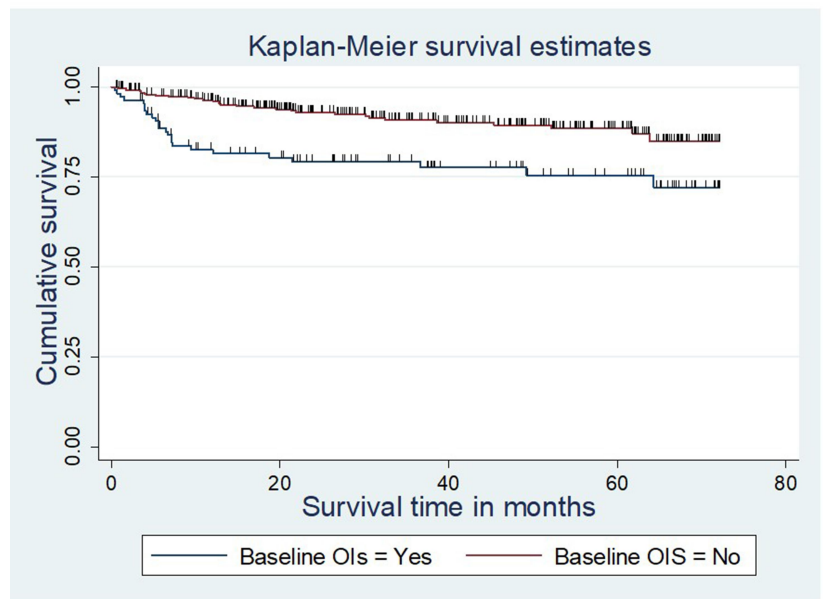

Figure 2 The Kaplan-Meier survival curves compare survival time of patients starting ART by baseline Ols in DBRH, North Showa, Amhara National Regional State, Ethiopia from January I, 2013 to December 30, 2018. dying thanthose who had stage I $(\mathrm{aHR}=2.88,95 \%$ $\mathrm{CI}=1.204-6.9$ ), and those who had stage IV had a 15.6-times higher risk of dying than those with stage I $(\mathrm{AHR}=15.6,95 \% \mathrm{CI}=6.61-36.94)$ (Table 4).

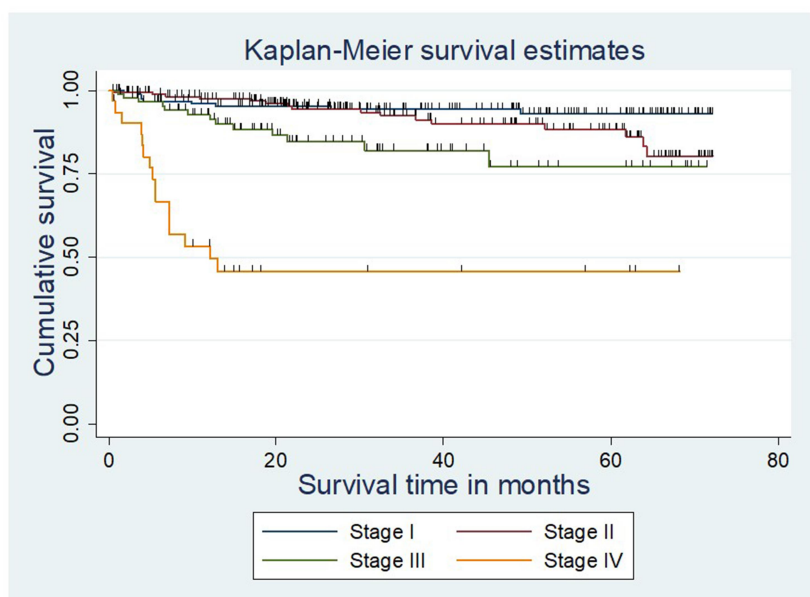

Figure 3 The Kaplan-Meier survival curves compare survival time of patients starting ART by baseline WHO clinical stage in DBRH, North Showa, Amhara National Regional State, Ethiopia from January I, 2013 to December 30, 2018. 


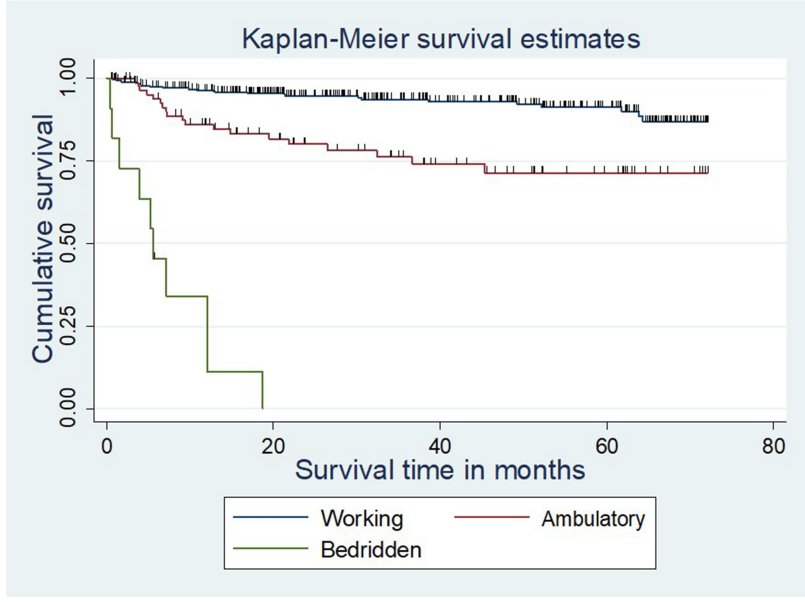

Figure 4 The Kaplan-Meier survival curves compare survival time of patients starting ART by functional status in DBRH, North Showa, Amhara National Regional State, Ethiopia from January I, 2013 to December 30, 2018.

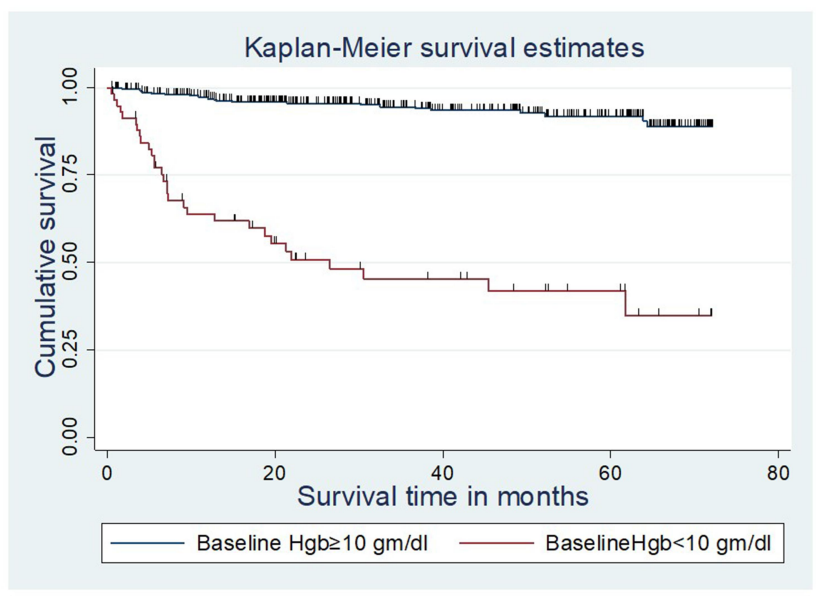

Figure 5 The Kaplan-Meier survival curves compare survival time of patients starting ART by baseline hemoglobin in DBRH, North Showa, Amhara National Regional State, Ethiopia from January I, 2013 to December 30, 2018.

\section{Discussion}

This retrospective cohort study was aimed to assess the survival and predictors of mortality among adult HIV/ AIDS patients who initiated highly active antiretroviral therapy. At the end of follow-up, 54 patients were dead and 393 patients were censored. That resulted in a cumulative incidence of deaths of $12.1 \%$ over 6 years. This finding is in line with studies conducted in the Somali Region, Eastern Ethiopia, which reported $11.1 \%,{ }^{13}$ and South Omo, Ethiopia, which reported $10 \% .{ }^{19}$ However, the total number of deaths that occurred in this study was higher than other studies conducted in southwest Ethiopia $(6.4 \%)^{22}$ and Northwest Ethiopia (4.2\%). ${ }^{8}$ But, some studies reported higher findings compared with this

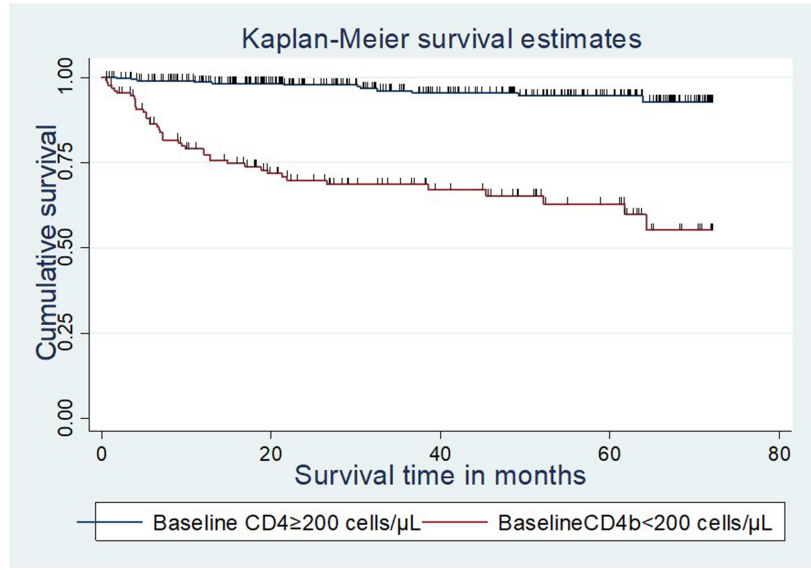

Figure 6

Figure 6 The Kaplan-Meier survival curves compare survival time of patients starting ART by baseline CD4 count in DBRH, North Showa, Amhara National Regional State, Ethiopia from January I, 2013 to December 30, 2018.

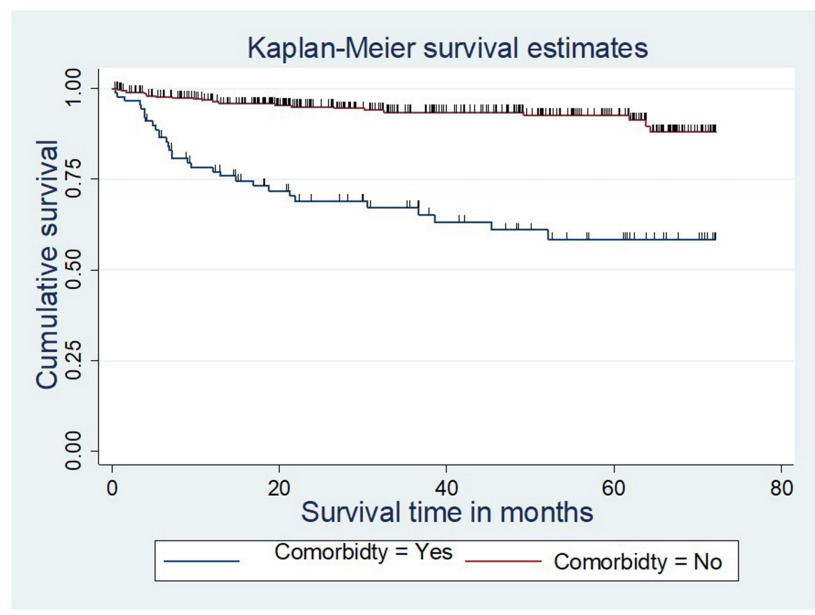

Figure 7 The Kaplan-Meier survival curves compare survival time of patients starting ART by comorbidity in DBRH, North Showa, Amhara National Regional State, Ethiopia from January I, 2013 to December 30, 2018.

study in the ART Center of MKCG Medical College, Berhampur, Ganjam, and Odisha $(21.33 \%)^{25}$ and Debre Markos (43\%). ${ }^{20}$ The discrepancy of results among studies might be due to differences in sample, in length of the study period, and differences in initiation of anti-retroviral therapy.

The current study revealed the overall mortality rate of patients on ART during 72 months follow-up was 4.18/ 100 person-years. This finding is in line with studies conducted by Dilla University Hospital, ${ }^{11}$ and seven university teaching hospitals in Ethiopia, ${ }^{12}$ where the incidence rates per person-year were 3.0 and 5.4, respectively. However, this finding is much higher than the findings reported in DebreMarkos Referral hospital, ${ }^{20}$ Jinka Hospital, South Omo Zone, 
Table 4 Results of the Bi-Variable and Multivariable Cox Regression Analyses of HIV Patients Receiving ART in DBRH ART Clinic, North Showa, Amhara National Regional State, Ethiopia, $2019(n=447)$

\begin{tabular}{|c|c|c|c|}
\hline Covariates & Category & $\begin{array}{l}\text { Bivariate cHR }(95 \% \\
\text { Cl) }\end{array}$ & $\begin{array}{l}\text { Multi-Variate aHR } \\
(95 \% \mathrm{Cl})\end{array}$ \\
\hline Marital status & $\begin{array}{l}\text { Single } \\
\text { Married } \\
\text { Divorced } \\
\text { Widowed }\end{array}$ & $\begin{array}{l}\text { I } \\
1.18(0.534-2.61) \\
2.826(1.17-6.82) \\
1.67(0.61-4.61)\end{array}$ & $\begin{array}{l}\text { I } \\
1.51(0.66-3.47) \\
3.475(1.41-8.55)^{b} \\
1.50(0.54-4.15)\end{array}$ \\
\hline Substance use & $\begin{array}{l}\text { Yes } \\
\text { No }\end{array}$ & $\begin{array}{l}1.75(1.01-3.04) \\
1\end{array}$ & $\begin{array}{l}1.59(0.84-2.98) \\
1\end{array}$ \\
\hline Disclosure & $\begin{array}{l}\text { Yes } \\
\text { No }\end{array}$ & $\begin{array}{l}\text { I } \\
1.80(0.99-3.27)\end{array}$ & $\begin{array}{l}\text { I } \\
1.24(0.61-2.5 I)\end{array}$ \\
\hline Past Ols & $\begin{array}{l}\text { Yes } \\
\text { No }\end{array}$ & $\begin{array}{l}2.58(1.51-4.43) \\
1\end{array}$ & $\begin{array}{l}1.86(1.05-3.33)^{\mathrm{a}} \\
1\end{array}$ \\
\hline TB treatment & $\begin{array}{l}\text { Yes } \\
\text { No }\end{array}$ & $\begin{array}{l}2.47(1.39-4.40) \\
1\end{array}$ & $\begin{array}{l}1.65(0.85-3.19) \\
1\end{array}$ \\
\hline CPT & $\begin{array}{l}\text { Yes } \\
\text { No }\end{array}$ & $\begin{array}{l}\mathrm{I} \\
2.32(1.28-4.21)\end{array}$ & $\begin{array}{l}1 \\
1.13(0.5-2.57)\end{array}$ \\
\hline Comorbidity & $\begin{array}{l}\text { Yes } \\
\text { No }\end{array}$ & $\begin{array}{l}5.97(3.48-10.24) \\
1\end{array}$ & $\begin{array}{l}2.56(1.39-4.74)^{b} \\
\text { । }\end{array}$ \\
\hline Baseline Hgb & $\begin{array}{l}\geq 10 \mathrm{mg} / \mathrm{dL} \\
<10 \mathrm{mg} / \mathrm{dL}\end{array}$ & $\begin{array}{l}\text { I } \\
11.75(6.85-20.15)\end{array}$ & $\begin{array}{l}\text { I } \\
4.7(2.25-9.62)^{\mathrm{c}}\end{array}$ \\
\hline Baseline CD4 & $\begin{array}{l}\geq 200 \text { cells/ } \\
\mu \mathrm{L} \\
<200 \text { cells/ } \\
\mu \mathrm{L}\end{array}$ & $\begin{array}{l}\text { I } \\
10.22(5.38-19.44)\end{array}$ & $\begin{array}{l}\text { I } \\
4.710(2.28-9.75)^{c}\end{array}$ \\
\hline $\begin{array}{l}\text { Functional } \\
\text { status }\end{array}$ & $\begin{array}{l}\text { Working } \\
\text { Ambulatory } \\
\text { Bedridden }\end{array}$ & $\begin{array}{l}\text { I } \\
3.545(1.950-6.443) \\
42.804(94.318)\end{array}$ & $\begin{array}{l}\text { I } \\
1.03(0.47-2.26) \\
3.07(1.11-8.480)^{\mathrm{a}}\end{array}$ \\
\hline $\begin{array}{l}\text { WHO clinical } \\
\text { staging }\end{array}$ & $\begin{array}{l}\text { Stage I } \\
\text { Stage II } \\
\text { Stage III } \\
\text { Stage IV }\end{array}$ & $\begin{array}{l}\text { I } \\
1.690(0.747-3.824) \\
3.334(1.420-7.827) \\
16.312(7.140-37.265)\end{array}$ & $\begin{array}{l}\text { I } \\
1.659(0.718-3.83 \mathrm{I}) \\
2.88(1.204-6.899)^{\mathrm{a}} \\
15.6(6.609-36.948)^{\mathrm{c}}\end{array}$ \\
\hline $\begin{array}{l}\text { ARV } \\
\text { adherence }\end{array}$ & $\begin{array}{l}\text { Good } \\
\text { Fair } \\
\text { Poor }\end{array}$ & $\begin{array}{l}\text { I } \\
4.419(2.134-9.151) \\
9.260(3.906-21.953)\end{array}$ & $\begin{array}{l}\text { I } \\
0.972(0.342-2.763) \\
2.366(0.759-7.377)\end{array}$ \\
\hline BMI & $\begin{array}{l}\leq 18.5 \\
18.5-25 \\
\geq 25\end{array}$ & $\begin{array}{l}6.764(2.058-22.232) \\
2.074(0.62 \mathrm{I}-6.932) \\
\mathrm{I}\end{array}$ & $\begin{array}{l}1.777(0.500-6.322) \\
1.449(0.423-4.969) \\
1\end{array}$ \\
\hline
\end{tabular}

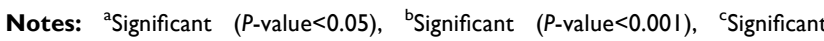
$(P$-value $<0.000$ I.

Ethiopia, ${ }^{19}$ and Jimma University Teaching Hospital, SouthWest Ethiopia, ${ }^{22}$ to which the mortality rates were reported as 1.9, 1.75, and 2.17 per person-years, respectively. The observed difference in mortality rates might be due to the difference in sample size, duration of the study period, and the time duration each cohort contributed.
The presence of opportunistic infection at the time of ART initiation was found to be an important predictor of survival. Accordingly, patients who have an opportunistic infection at ART initiation are 1.86-times more likely to die as compared to those who do not have an opportunistic infection on ART initiation. Studies conducted at Fiche hospital in North Showa, Oromia region, Ethiopia, a selected public hospital in Harar, eastern Ethiopia, and India supported that the presence of opportunistic infection increases the probability of death. ${ }^{16,26,27}$ This might be because opportunistic infections may increase the risk of mortality in the first few months after initiating ART due to immune reconstitution syndrome, a paradoxical worsening or recurrence of opportunistic infection symptoms as a result of the rapid immunological recovery. ${ }^{28}$

The current study revealed that patients with baseline WHO clinical stage III and IV were 2.88-times and 15.6-times more likely to die compared to patients with WHO clinical stage I or II, respectively. Different studies supported this idea. ${ }^{20,27}$ This might be due to the fact that patients died mostly because of their late initiation of ART when they had the worst health condition. Early initiation before the advancement of the disease is recommended as soon as the confirmation of HIV diagnosis, which helps decrease the morbidity and mortality of HIV/AIDS. Besides, people with advanced HIV disease require closer follow-up during the initial period of receiving ART to monitor the response to ART and to identify signs and symptoms of possible immune reconstitution inflammatory syndrome.

This study showed that patients who were bedridden at ART initiation were 3.069-times more likely to die compared to the patients with working functional status at treatment initiation. This finding is supported by the study conducted in Jimma university teaching hospital, Khartoum State, Sudan, and Eastern Ethiopia in which bedridden patients were 2.5-, 4.765-, and 4.09-times more likely to die than those patients with working and ambulatory functional status, respectively. ${ }^{22,29,30}$ This could be due to poor immunologic response, mainly failure to restore CD4 cells to 500 cells $/ \mu \mathrm{L}$ despite being virally suppressed.

This study indicated that the baseline hemoglobin level $<10 \mathrm{gm} / \mathrm{dL}$ was found to be a significant predictor of death among HIV positive patients on ART. Accordingly, patients who had a hemoglobin level $<10 \mathrm{gm} / \mathrm{dL}$ were 4.7-times more likely to die as compared to those who had hemoglobin levels greater than or equal to $10 \mathrm{gm} / \mathrm{dL}$. This finding is supported by previous studies that have been conducted in Aksum, Northern Ethiopia. ${ }^{9}$ Maybe 
because anemia is one of the markers of advanced disease and can increase the mortality of patients.

This study revealed that patients who had a CD4 count $<200$ cells $/ \mu \mathrm{L}$ were found to be 4.7 -times more likely to die than to those who have a CD4 count $\geq 200$ cells/ $\mu \mathrm{L}$. A study conducted in Jimma university teaching hospital indicated that patients who were on ART with CD4 $<200$ cells $/ \mu \mathrm{L}$ were 1.3 -times more likely to die when compared to those whose CD4 was $\geq 200$ cells $/ \mu \mathrm{L}^{22}$

This discrepancy might be due to a difference in sample size and the eligible age groups for the study. Above all, the reduction of CD4 count decreases the immunity of patients, exposing them to opportunistic infections and predispose to micronutrient deficiency like iron which in turn causes the reduction of the amount of hemoglobin, which was an important predictor for mortality of patients initiated ART. Moreover, PLWH who have a CD4 cell count below 200 are at high risk of developing serious illnesses.

The current study showed that patients who had comorbidity have a 2.56-times higher hazard of mortality than those who had no comorbidity. This could be due to the fact that comorbidities complicate caring infrastructures for HIV-infected patients, because patients with multiple diseases and on complex pharmacological treatments are particularly difficult to manage, both in chronic and acute phases.

\section{Limitation of the Study}

Selection bias was possibly introduced due to the fact that patients with incomplete records of variables or charts which were lost for some patients were excluded. Therefore, those study subjects whose charts were not included in the study and with missing values may undermine the result if it is related with death.

\section{Conclusion}

In the current study, a total of 54 (12.1\%) patients on ART died during the follow-up period. Hence, the overall incidence rate was 4.18 per 100 -person years with an overall mean survival time of 63.7 months. Close follow-up and monitoring should be given to patients who have baseline $\mathrm{CD} 4<200$ cells $/ \mu \mathrm{L}$, opportunistic infections at baseline, patients with hemoglobin $<10 \mathrm{gm} / \mathrm{dL}$, functional status of bedridden, and those who have comorbidity. Special emphasis and close follow-up should be given to patients who had baseline stage III and IV disease. Further predictors of mortality of patients on ART needs to be studied with another strong (prospective) study design.

\section{Abbreviations}

AAU, Addis Ababa University; AIDS, Acquired Immune Deficiency Syndrome; AMA, American Medical Association; ART, Antiretroviral Therapy; CI, Confidence Interval; CPT, Cotrimoxazole Prophylactic Therapy; DB, Debre Berhan; DBRH, Debre Berhan Referral Hospital; EPHI, Ethiopian Public Health Institute; HAART, Highly Active Antiretroviral Therapy; HGB, Hemoglobin; HIV, Human Immunodeficiency Virus; HR, Hazard Ratio; IQR, Interquartile Range; $\mathrm{Mg} / \mathrm{dL}$, Milligram per Deciliter.

\section{Data Sharing Statement}

Since this is funded research, the raw data can be obtained from the investigators upon reasonable formal request.

\section{Ethics Approval and Consent to Participate}

Ethical clearance had been obtained from the Institutional Review Board (IRB) of Addis Ababa University, College of Health Science before conducting the study. After securing ethical clearance from IRB of Addis Ababa University, Debre Berhan Referral Hospital had been informed about the objective of the study through a support letter from Addis Ababa University, School of nursing and midwifery. This study was conducted in accordance with the Declaration of Helsinki (IRB protocol No:020/19/SNM; Institute: AAU, CHS, School of Nursing and Midwifery). Informed consent was not needed since the study was conducted through a review of medical records; the individual patients had not been exposed to any harm as far as the confidentiality was kept. To keep the confidentiality of the patients, data collectors were recruited from ART clinic and personal identifiers had not been included in the data collection format.

\section{Acknowledgments}

The authors are grateful to the college of health sciences, Addis Abeba University for funding the project. The authors would like to express their special thanks to all data collectors.

\section{Author Contributions}

All authors made a significant contribution to the work reported, whether that is in the conception, study design, execution, acquisition of data, analysis and interpretation, or in all these areas; took part in drafting, revising, or 
critically reviewing the article; gave final approval of the version to be published; have agreed on the journal to which the article has been submitted; and agree to be accountable for all aspects of the work.

\section{Funding}

This work was fully funded by Addis Ababa University. The funder has no role in the design of the study, data collection, and analysis, interpretation of data, and writing the manuscript.

\section{Disclosure}

The authors declare that they have no competing interests.

\section{References}

1. UNAIDS. 2017 Global HIV Statistics; 2018.

2. UNAIDS. Global Statistics of Hiv/Aids; 2017.

3. EPHI. HIV Related Estimates and Projections for Ethiopia; 2017.

4. WHO. Global Action Plan on Hiv Drug Resistance 2017-2021: 2018 Progress Report; 2018.

5. UNAIDS. Global AIDS Onitoring 2019 Indicators for Monitoring the 2016 Political Declaration on Ending AIDS; 2018.

6. FMOH. Guide for Implementation of Antiretroviral Therapy Program in Ethiopia, Federal HIV/AIDS Prevention and Control Office; 2007.

7. Biset Ayalew M. Mortality and its predictors among hiv infected patients taking antiretroviral treatment in Ethiopia: a systematic review. AIDS Res Treat. 2017;2017:1-10. doi:10.1155/2017/5415298

8. Assemie MA, Muchie KF, Ayele TA. Incidence and predictors of loss to follow up among HIV-infected adults at Pawi General Hospital, northwest Ethiopia: competing risk regression model. BMC Res Notes. 2018;11(1):287.

9. Tadesse K, Haile F, Hiruy N, Sued O. Predictors of mortality among patients enrolled on antiretroviral therapy in Aksum hospital, northern Ethiopia: a retrospective cohort study. PLoS One. 2014;9(1): e87392. doi:10.1371/journal.pone.0087392

10. Mengesha S, Belayihun B, Kumie A. Predictors of Survival in HIVInfected Patient After Initiation of HAART in Zewditu Memorial Hospital, Addis Ababa, Ethiopia. International scholarly research notices; 2014.

11. Hailemariam S. Determinants of Survival in HIV Patients: A Retrospective Study of Dilla University Hospital HIV Cohort. 2016.

12. Fekade D, Weldegebreal T, Teklu AM, et al. Predictors of survival among adult Ethiopian patients in the national ART program at Seven University Teaching Hospitals: a prospective cohort study. Ethiop J Health Sci. 2017;27(1):63-71. doi:10.4314/ejhs.v27i1.7S

13. Damtew B, Mengistie B, Alemayehu T. Survival and determinants of mortality in adult HIV/Aids patients initiating antiretroviral therapy in Somali Region, Eastern Ethiopia. Pan Afr Med J. 2015;22:138. doi:10.11604/pamj.2015.22.138.4352

14. Kebebew K, Wencheko E. Survival analysis of HIV-infected patients under antiretroviral treatment at the armed forces general teaching hospital, Addis Ababa, Ethiopia. Ethiop J Health Dev. 2012;26 (3):186-192.
15. Seyoum D, Degryse J-M, Kifle Y, et al. Risk factors for mortality among adult HIV/AIDS patients following antiretroviral therapy in Southwestern Ethiopia: an assessment through survival models. Int J Environ Res Public Health. 2017;14(3):296. doi:10.3390/ ijerph14030296

16. Tesfamariam K, Baraki N, Kedir H. Pre-ART nutritional status and its association with mortality in adult patients enrolled on ART at Fiche Hospital in North Shoa, Oromia region, Ethiopia: a retrospective cohort study. BMC Res Notes. 2016;9(1):512. doi:10.1186/s13104-016-2313-y

17. Tadege M. Time to death predictors of HIV/AIDS infected patients on antiretroviral therapy in Ethiopia. BMC Res Notes. 2018;11 (1):761. doi:10.1186/s13104-018-3863-y

18. Refera H, Wencheko E. Survival of HIV-TB co-infected adult patients under ART in Ambo Referral Hospital, Ethiopia. Ethiop $J$ Health Dev. 2013;27(2):88-93.

19. Tachbele E, Ameni G. Survival and predictors of mortality among human immunodeficiency virus patients on anti-retroviral treatment at Jinka Hospital, South Omo, Ethiopia: a six years retrospective cohort study. Epidemiol Health. 2016;38.

20. Tadele A, Shumey A, Hiruy N. Survival and predictors of mortality among adult patients on highly active antiretroviral therapy at Debre-Markos Referral Hospital, North West Ethiopia; a Retrospective Cohort Study. J AIDS Clin Res. 2014;5(280):2.

21. AIHA. How Twinning Helped Transform Debre Berhan Referral Hospital into a National Model for Quality Care and Effective Management. The Debre Berhan/Elmhurst Hospital Center Partnership: a Successful Model for Global Health and Development; 2014.

22. Gesesew HA, Ward P, Woldemichael K, Mwanri L, De Socio GV. Early mortality among children and adults in antiretroviral therapy programs in Southwest Ethiopia, 2003-15. PLoS One. 2018;13(6): e0198815. doi:10.1371/journal.pone.0198815

23. CDC. Epi Info ${ }^{\mathrm{TM}}$ version 7.1.2.0. 2013

24. Zhang Z. Model building strategy for logistic regression: purposeful selection. Ann Transl Med. 2016;4(6).

25. Benu P, Rao SHB, Ramachandra M, Simanchal P, Prasanna K. Predictors of mortality among HIV patients on HAART in an ART centre-a Retrospective Study. Int J Med Sci Public Health. 2016;6(4).

26. Joseph N, Sinha U, Tiwari N, Ghosh P, Sindhu P. Prognostic factors of mortality among adult patients on antiretroviral therapy in India: a Hospital Based Retrospective Cohort Study. Biomed Res Int. 2019;2019:1-10. doi:10.1155/2019/1419604

27. Digaffe T, Seyoum B, Oljirra L. Survival and predictors of mortality among adults on antiretroviral therapy in Selected Public Hospitals in Harar, Eastern Ethiopia. J Trop Dis. 2014;2(148):2. doi:10.4172/ 2329-891X.1000148

28. Sharma SK, Soneja M. HIV \& immune reconstitution inflammatory syndrome (IRIS). Indian $J$ Med Res. 2011;134(6):866-877. doi:10.4103/0971-5916.92632

29. Sato S, Masui K, Nishina N, et al. Initial predictors of poor survival in myositis-associated interstitial lung disease: a multicentre cohort of 497 patients. Rheumatology. 2018;57(7):1212-1221. doi:10.1093/ rheumatology/key060

30. Biadgilign S, Reda AA, Digaffe T. Predictors of mortality among HIV infected patients taking antiretroviral treatment in Ethiopia: a retrospective cohort study. AIDS Res Ther. 2012;9(1):15. doi:10.1186/1742-6405-9-15 


\section{Publish your work in this journal}

HIV/AIDS - Research and Palliative Care is an international, peerreviewed open-access journal focusing on advances in research in $\mathrm{HIV}$, its clinical progression and management options including antiviral treatment, palliative care and public healthcare policies to control viral spread. The manuscript management system is completely online and includes a very quick and fair peer-review system, which is all easy to use. Visit http://www.dovepress.com/testimonials.php to read real quotes from published authors.

Submit your manuscript here: https://www.dovepress.com/hivaids—research-and-palliative-care-journal 